\title{
Hepatic infarction in a pregnant woman with antiphospholipid syndrome and early pre-eclampsia: A case report
}

\author{
Lampros Ntovas ${ }^{1}$, Athanasios Moles ${ }^{1}$, Nektaria Papadatou ${ }^{1}$, Eugenia Kourakou ${ }^{1}$, Kiriakos Mazis ${ }^{1}$, Leonidas \\ Grigorakos*2 \\ ${ }^{1}$ Rea Maternity Hospital, Athens, Greece \\ ${ }^{2}$ National and Kapodistrian University of Athens, Athens, Greece
}

Received: March 27, 2020

DOI: $10.5430 /$ crim.v7n2p18
Accepted: May 24, 2020

Online Published: June 23, 2020

URL: https://doi.org/10.5430/crim.v7n2p18

\begin{abstract}
The aim of the present case report is to stress out the occurrence of hepatic infraction in pregnant women with antiphospholipid syndrome (APS) and the critical role magnetic resonance imaging (MRI) plays for the early diagnosis of rare complications of the disease. Here we report the case of a preeclamptic pregnant woman with APS in her 17th week of gestation, who experienced severe epigastric pain, followed by multiple episodes of vomiting and an episode of soft stools. Patient was submitted to MRI examination of the upper abdomen, where findings suggested hepatic infarction with subsequent deterioration of her general status. Therefore, she underwent termination of gestation. We conclude that pregnancy is a deteriorating factor of the disease and patient should be closely monitored, evaluated of any suspicious symptom or sign.
\end{abstract}

Key Words: Antiphospholipid syndrome, Hepatic infarction, MRI, Pregnancy management

\section{INTRODUCTION}

Antiphospholipid syndrome (APS) is an autoimmune disorder, characterized by the presence of a variety of autoimmune antibodies (e.g. anticardiolipin antibody, B2- glycoprotein antibody, lupus anticoagulant). ${ }^{[1,2]}$ These antibodies replace the protective anticoagulant proteins of the phospholipid membranes, leading to the production of procoagulant endothelial cell surfaces. Thus, the syndrome is accompanied by arterial and venous thrombotic events and obstetric complications (early onset pre-eclampsia, repeated pregnancy loss and preterm delivery, uteroplacental insufficiency leading to fatal growth restriction). ${ }^{[3-5]}$ The APS appears to $0.3 \%$ of the pregnant population. Patients with APS are in higher risk to develop pre-eclampsia than the general population; a recent metanalysis shows that the presence of APS increases the incidence of pre-eclampsia twice compared to the healthy pregnant woman. The most significant fact is that the incidence of pre-eclampsia is highly increased (eleven times) till the 34th week of gestation, while after this point is remarkably less, and presents in more severe form. ${ }^{[6,7]}$ Abdominopelvic manifestations of thromboembolism in APS patients are rarely reported in the literature and mostly present as Budd-Chiari syndrome. The risk of arterial and venous thrombotic events appears to be 5\%-12\% in pregnant women with APS. ${ }^{[8]}$ The frequency of repeated pregnancy loss varies. However, studies reveal that $15 \%$ of Greece.

*Correspondence: Leonidas Grigorakos; Email: grigorakos@parliament.gr; Address: National and Kapodistrian University of Athens, Athens, 
women with pregnancy loss in the first trimester had APS autoantibodies while the incidence increased to $21 \%$ in the second trimester. ${ }^{[9]}$ Hepatic infarction is a very rare event because of hepatic distinct double blood supply. Here, we report on a pregnant woman with APS on the 17th week of gestation.

\section{CASe presentation}

A 35-year-old childless woman, at 17th week of gestation (17 week and 5 days), with a history of APS under treatment with Bemiparin sodium 5,000 IU by sc route, referred to emergency room (ER) with abdominal pain in the epigastrium started $18 \mathrm{~h}$ ago followed by multiple episodes of vomiting (08) and an episode of soft stools. The history revealed Deep Vein Thrombosis (DVT) in 2005 which was followed by the diagnosis of APS, an episode of fetal resorption, therapy with Tinzaparin 8,000 IU by sc route and acetylsalicylic acid 160 mg p.os till the beginning of pregnancy with recommendation, by the referred hematologist, of checking anti-factor $\mathrm{X} \alpha$ followed by changing to Bemiparin sodium 5,000 IU by sc route. The patient, before coming to the emergency room, had already received p.os Paracetamol /Butylscopolamine bromide $(500+10) \mathrm{mg}$ and p.os Omeprazole $20 \mathrm{mg}$ without restriction of symptoms. At the emergency room the patient presented temperature $37.2^{\circ} \mathrm{C}$, BP120/70 $\mathrm{mmHg}$, cardiac beats 57 BPM, $\mathrm{SO}_{2} 98 \%$, abdominal tenderness at the epigastrium, positive Murphy sign, diminished bowel sounds and dehydrated mucous membranes. The patient underwent biochemical and ultrasound examination which revealed:

- Increased transaminases and Lactate Dehydrogenase (LDH): Aspartate Aminotransferace (AST) 120 IU/lt Alanine Aminotransferace ALT138 IU/lt LDH 313 IU/lt, Newly developed proteinuria ( 24 hours urine protein $500 \mathrm{mg}$ ).

- Ultrasound (U/S) of the abdomen without significant findings, normal Doppler of the hepatic veins. The diagnostic dilemma was still pending while the patient's abdominal pain deteriorated. Therefore, we proceeded to MRI of the upper abdomen (without using iv contrast media) that showed multiple hepatic infarcts (at segment V, between segments V and VI, at segment VIII and at the inner part of segment VI and bilateral pleural effusion) (see Figure 1).

The patient was transferred to the Intensive Care Unit (ICU), where she deteriorated hemodynamically; the BP increased to $170 / 85 \mathrm{mmHg}$ and the $\mathrm{SO}_{2}$ decreased to $92 \%$ (ambient air). She underwent transthoracic echocardiogram which revealed Pulmonary Artery Systolic Pressure (PASP) 40$45 \mathrm{mmHg}$, whole leg ultrasonography without important findings and new biochemical examination which revealed further increase in transaminases and LDH:
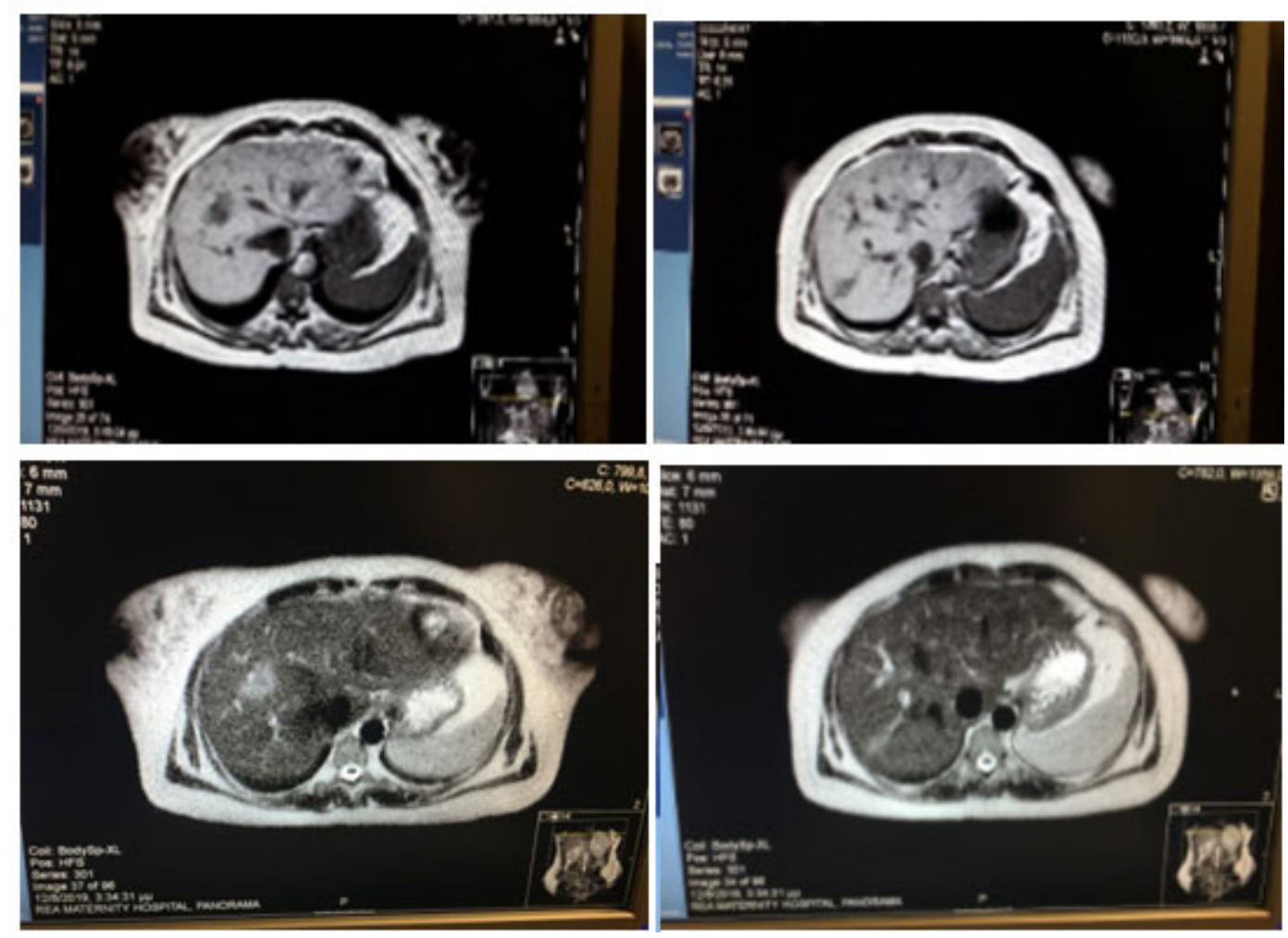

Figure 1. MRI Findings - Multiple lesions in the right lobe of the liver, hypointense on T1 and hyperdense on T2 imaging 
- AST 258 IU/lt ALT 322 IU/lt LDH 402 IU/lt. The patient was placed to hemodynamic monitoring, nasal canula $\mathrm{O}_{2}$ at $3 \mathrm{lt} / \mathrm{min}$, iv fluids, iv painkillers, iv antihypertensive drugs and Bemiparin sodium 5,000 IU by sc route.

The next day a new biochemical profile revealed:

- AST 400 IU/lt ALT 506 IU/lt LDH 442 IU/lt. The general status of the patient was deteriorating and so did her kidney function (Creatinine $1.4 \mathrm{mg} / \mathrm{dl}$ from $0.9 \mathrm{mg} / \mathrm{dl}$ ).

The probability of amniotic fluid embolism occurrence was eliminated as a picture of circulatory failure (shock), respiratory failure and bleeding was absent. More so differential diagnosis for complicated gallstone disease, viral and other vascular diseases (including Budd Chiari syndrome) was performed and subsequently diseases were excluded.

However, because of the overall deterioration, a decision to terminate gestation was made. We used Sopher forceps for dilation and then suction curettage was performed. Twelve hours after the procedure, we readministered Bemiparin sodium 5,000 IU by sc route. Twenty-four hours later, her epigastric pain had subsided. Her AST/ALT levels were lower (150/240 IU/L). In addition, LDH was $250 \mathrm{IU} / \mathrm{L}$ and the platelet count was $180,000 / \mathrm{ml}$.

On the second day, pulmonary embolism was excluded by performing CT angiography. Seven days after the termination of pregnancy, her epigastric pain was completely alleviated and she was discharged with anticoagulant treatment. A liver CT 4 weeks after discharge revealed that the extent of infarction had decreased.

\section{DisCussion}

Venous stasis, alteration of thrombotic factors and the fibrinolytic system provoke a hypercoagulable tendency in pregnant women. These unique characteristics of pregnancy aggravate the risk of thromboembolism in pregnant women with APS. ${ }^{[5,10]}$ Thromboses are the hallmark of APS. Deep veins of the lower extremities are the most common sites of venous thrombosis, and the cerebral vasculature is the most common site for arterial thrombosis.

In prospective studies, the risk of thromboembolic disease during pregnancy or postpartum was $5 \%$ to $12 \%$ among women with known APS, compared with $0.025 \%$ to $0.10 \%$ in the general obstetric population. Women with thrombosisassociated APS have higher rates of pregnancy complications/fetal loss than those with only obstetric-associated APS. Conventional treatment with low dose aspirin/low molecular weight heparin of patients with obstetric APS fails to prevent morbitity in $20 \%$ of cases. There is no second line therapy for women with adverse pregnancy outcomes despite antithrombotic therapy. ${ }^{[11]}$

Liver dysfunction with abnormal liver tests in pregnancy represents $3 \%$ of the deliveries. The cardinal features of hepatobiliary disease can include jaundice, pruritus, abdominal pain, nausea, vomiting, and a variety of liver biochemical test abnormalities. Because the liver has a dual blood supply, hepatic infarction is a very uncommon and life-threatening complication and its early diagnosis is quite difficult. It results from occlusion of a single intrahepatic branch of the hepatic artery. Hepatic artery branches to the right hepatic lobe are affected most commonly. Many causes of hepatic infarction have been reported. Hepatic infarction associated with toxemia of pregnancy is notable for its tendency to cause hepatic hemorrhage, which may lead to rupture of Glisson's capsule and catastrophic intraabdominal bleeding. ${ }^{[12]}$

Hepatic infarction may not cause symptoms or may cause fever, epigastric or right upper quadrant pain, back or right shoulder pain, jaundice, nausea, and vomiting. A marked leukocytosis is common. The aminotransferases rise transiently and occasionally to massive levels. Computed tomography is more sensitive compared with ultrasound for detecting a focal hepatic lesion. On MRI, hepatic infarction typically appears as a wedge-shaped region of diminished T1 signal and increased T2 signal, a finding nonspecific. Doppler ultrasonography should be performed to assess the patency of the hepatic artery. However, the use of MRI in pregnancy is very helpful and as it is supported by the literature, it is safe for both the mother and the fetus. ${ }^{[13]}$ Literature reveals no difference in terms of exposure to MRI during the first trimester of pregnancy, concluding that it does not produce damage to the fetus. Opposingly, when gadoliniumbased MRI contrast agents are used during pregnancy, they can cross the placenta and enter the fetal circulation, thus leading to stillbirth or even fetus' death. Consequently, performing MRI in pregnant patients should be assessed on a case-to-case basis, while the use of gadolinium-based MRI contrast agents should be avoided in any case. ${ }^{[14]}$

To conclude, the pathogenic mechanisms which are involved in APS are various and thus the patients present a great variability of clinical manifestations. Pregnancy is a deteriorating factor of the disease and the patient should be closely monitored, evaluated of any suspicious symptom or sign. The role of MRI is critical for the early diagnosis of rare complications of the disease, such hepatic infarctions are.

\section{CONFLICTS OF INTEREST DisClosure}

The authors have declared no conflicts of interest. 


\section{REFERENCES}

[1] Vitoratos N, Avarantinos L. Antiphospholipid syndrome and pregnancy. Perigenetic Medicine and Neonatology. 2011; 6(2): 35-43 (in Greek).

[2] Paulzner R, Dulitzky M, Carp H, et al. Hepatic infarctions during pregnancy are associated with the antiphospholipid syndrome and in addition with complete or incomplete HELLP syndrome. Journal of Thrombosis and Haemostasis. 2003; 1: 1758-1763. PMid:12911590. https://doi.org/10.1046/j.1538-7836.2003.00253.x

[3] Lazzaroni MG, Fredi M, Andreoli L, et al. Triple Antiphospholipid (aPL) Antibodies Positivity is Associated with Pregnancy Complications in aPL Carriers: A Multicenter Study on 62 Pregnancies. Front Immunol. 2019 Aug 14; 10: 1948. PMid:31475009. https://doi.org/10.3389/fimmu . 2019.01948

[4] Guo Q, Yang Z, Guo J, et al. Hepatic infarction induced by HELLP syndrome: a case report and review of the literature. BMC Pregnancy and Childbirth. 2018; 18: 191. PMid:29848304. https: //doi.org/10.1186/s12884-018-1799-9

[5] Rohan W, Pierangeli S. Pathophysiology of the antiphospholipid antibody syndrome. Auto Immun Highlights. 2011 Nov; 2(2): 3552. PMid:26000118 . https://doi.org/10.1007/s13317-011 $-0017-9$

[6] Marchetti T, de Moerloose P, Gris JC. Antiphospholipid antibodies and the risk of severe and non-severe pre-eclampsia: the NOHA casecontrol study. J Thromb Haemost. 2016; 14: 675-84. PMid:26782635. https://doi.org/10.1111/jth.13257

[7] Kim JH, Yee C, Kuk JY, et al. Hepatic infarction in the pregnant woman with antiphospholipid syndrome and triple antibody positivity: A case report focusing on catastrophic antiphospholipid syndrome. Obstet Gynecol Sci. 2016; 59(5): 397-402. PMid:27668204. https://doi.org/10.5468/ogs.2016.59.5.397
[8] Navarro CD, Del Carmen Castillo-Hernandez M, Majluf-Cruz K, Espejo-Godinez G, Carmona-Olvera P, Moreno-Hernandez M, LugoGarcía Y, Hernandez-Juarez J, Loarca-Piña L, Isordia-Salas I, MajlufCruz A. Impact of Classical Risk Factors for Arterial or Venous Thrombosis in Patients with Antiphospholipid Syndrome. Clin Appl Thromb Hemost. 2018 Jul; 24(5):834-840. PMid:28884609. https : //doi.org/10.1177/1076029617727859

[9] Di PF, Valenti O, Hyseni E, et al. Antiphospholipid Syndrome during pregnancy: the state of the art. J Prenat Med. 2011 Apr; 5(2):41-53.

[10] Gomez-Puerta JA, Cervera R, Espinosa G, et al. Catastrophic antiphospholipid syndrome during pregnancy and puerperium: maternal and fetal characteristics of 15 cases. Ann Rheum Dis. 2007; 66: 740-746. PMid:17223653. https://doi.org/10.1136/ard. 20 06.061671

[11] Galli M. Treatment of the antiphospholipid syndrome. Auto Immun Highlights. 2013 Dec 22; 5(1): 1-7. PMid:26000150. https : //doi.org/10.1007/s13317-013-0056-5

[12] Troja A, Abdou A, Rapp C, et al. Management of Spontaneous Hepatic Rupture on Top of HELLP Syndrome: Case Report and Review of the Literature. Viszeralmedizin. 2015 Jun; 31(3): 205-8. PMid:26468317. https ://doi.org/10.1159/000376601

[13] Ray JG, Vermeulen MJ, Bharatha A, et al. Association Between MRI Exposure During Pregnancy and Fetal and Childhood Outcomes. JAMA. 2016; 316(9): 952-961. PMid:27599330. https : //doi.org/10.1001/jama.2016.12126

[14] UCSF Department of Radiology and Biomedical Imaging, CT and MR Pregnancy Guidelines. Available at https ://radiology .uc sf .edu/patient-care/patient-safety/ct-mri-pregnan cy\#accordion-pregnancy-and-mri-inpatients 